\title{
Congestion Behavior under Uncertainty on Morning Commute with Preferred Arrival Time Interval
}

\author{
LingLing Xiao, ${ }^{1}$ Ronghui Liu, ${ }^{2}$ and HaiJun Huang ${ }^{1}$ \\ ${ }^{1}$ School of Economics and Management, Beihang University, Beijing 100191, China \\ ${ }^{2}$ Institute for Transport Studies, University of Leeds, Leeds LS2 9JT, UK \\ Correspondence should be addressed to Ronghui Liu; r.liu@its.leeds.ac.uk
}

Received 5 November 2013; Accepted 18 December 2013; Published 17 February 2014

Academic Editor: Huimin Niu

Copyright (C) 2014 LingLing Xiao et al. This is an open access article distributed under the Creative Commons Attribution License, which permits unrestricted use, distribution, and reproduction in any medium, provided the original work is properly cited.

This paper extends the bottleneck model to study congestion behavior of morning commute with flexible work schedule. The proposed model assumes a stochastic bottleneck capacity which follows a uniform distribution and homogeneous commuters who have the same preferred arrival time interval. The commuters are fully aware of the stochastic properties of travel time and schedule delay distributions at all departure times that emerge from day-to-day capacity variations. The commuters' departure time choice follows user equilibrium (UE) principle in terms of the expected trip cost. Analytical and numerical solutions of this model are provided. The equilibrium departure time patterns are examined which show that the stochastic capacity increases the mean trip cost and lengthens the rush hour. The adoption of flexitime results in less congestion and more efficient use of bottleneck capacity than fixed-time work schedule. The longer the flexi-time interval is, the more uniformly distributed the departure times are.

\section{Introduction}

The bottleneck model was first proposed by Vickrey [1] and has subsequently inspired many to develop more realistic extensions, to gain qualitative and theoretical insights into transport policy measures on commuter travel behavior (e.g., Smith [2]; Daganzo [3]; Braid [4]; Arnott et al. [5]; Yang and Huang [6]; Ramadurai et al. [7]). In these analyses, commuters must choose their departure times to minimize the sum of travel delays (depending on the bottleneck capacity) and schedule delays (depending on the difference between actual and desired arrival times). At user equilibrium, no commuter could reduce the total commute cost by unilaterally changing his/her departure time.

The study of decision making under risk (and uncertainty) has a long history in the fields of economics, psychology, transport, and beyond (Machina [8]; Cubitt and Sugden [9]; Gollier and Treich [10]; Birnbaum and Schmidt [11]). Many mathematical tools and analytical frameworks are used to model people's behavior and predict likely choice outcomes in varying settings. The theory has also evolved from expected utility to nonexpected utility and been used to describe choice behavior in risky situations (Starmer [12]; de Palma et al. [13]).
This paper applies the expected utility theories to capture the departure time choice behavior in morning commute problem under uncertainty. Morning commute plays an important role in a monocentric city and the traffic congestion in such network is caused by concentration of travel demand around the work start time. The introduction of flexible work schedule is one of the transport demand management measures for alleviating peak congestion. The paper provides useful insight into traveler's decision making in contrast to fixed-time schedule pattern. Henderson [14] incorporated the productivity effect to analyze the equilibrium and optimum solution with staggered work hours. Mun and Yonekawa [15] extended bottleneck congestion to study the case that part of the firms in city adopts flexitime and incorporated the effects on urban productivity.

Most literatures on morning commute have assumed that the capacity of the bottleneck is deterministic and the traffic demand is also deterministic or governed by a predetermined elastic demand function. In reality, not only does travel time increase as traffic volume increases towards capacity but also the travel time becomes increasingly random and unpredictable due to the chaotic behavior of traffic at the micro level. The source of variation in road capacity may 
occur due to physical and operational factors, such as road repairs, construction, accidents, and bad weather. The variations in road capacity from physical and operational reasons are what make the analysis of travel behavior so complex and yet interesting. As such, understanding travelers' attitudes and their behavior in varying settings is key to developing sustainable transport polices. There has been recent attention to the stochastic nature of the bottleneck models (Siu and Lo [16]; Li et al. [17]; Xiao et al. [18]). A reasonable way to capture these variations and their impact on the network performance is to formulate the problem using probability distributions (Chen et al. [19]).

The focus of this paper is to analyze the departure time choice behavior under uncertainty in the morning commuting problem with flexi-time work schedule. It is expected that the stochastic capacity leads to uncertainty in queuing, travel time, and trip cost, which in turn influences the commuters' travel choice behavior. We assume that travelers are fully aware of the stochastic properties of the travel time and schedule delay distributions throughout the morning peak period which emerges from their day-to-day travel experience. Furthermore, we consider homogenous travelers have the same preferred arrival time interval (PATI). We formulate a stochastic bottleneck model for this flexi-time commute problem and derive its analytical solution. The properties of the model are investigated.

The solution of the proposed model shows that the capacity variability of the bottleneck leads to significant changes in departure time patterns, which are different to those derived under deterministic conditions. In a deterministic bottleneck model with flexi-time work schedule, an individual can choose either to depart in the tails of the rush hour when travel time is low and pay the penalty of arriving at work early or late, or to depart close to the PATI when travel time is high but schedule delay cost is low. In other words, under the deterministic equilibrium, schedule delay early/late and arrival on time cannot occur simultaneously for a given departure time (Henderson [14]; Mun and Yonekawa [15]). We demonstrate that, with day-to-day stochastic capacity, commuters departing at the same time may endure schedule delay early/late or not and may experience queuing delay or not on different days.

The rest of this paper is organized as follows. Section 2 formulates separately a deterministic and a stochastic bottleneck model with homogeneous PATI. The equilibrium solutions for the departure time pattern are derived for each case. The theoretical properties of the proposed stochastic bottleneck model with PATI are investigated and compared with the deterministic case. Numerical examples are presented in Section 3 to illustrate further the equilibrium properties of the model. Section 4 provides conclusion remarks.

\section{Departure Patterns of Morning Commute with Flexible Arrival Time}

2.1. The Deterministic Case. We formulate the peak period congestion based on the bottleneck model developed by Vickrey [1]. Suppose a single road connecting a residential area and the Commercial Business District (CBD), which has a bottleneck just before the CBD. It is assumed that vehicles drive at constant speed from home to the bottleneck point: travel time for this portion of trip is constant and represented as $T_{\text {free }}$. Queue develops when traffic flow rate exceeds the bottleneck capacity $s$. Travel time for a vehicle departing at time instant $t$ is represented as follows:

$$
T(t)=T_{\text {free }}+\frac{Q(t)}{s},
$$

where $T(t)$ is travel time and $Q(t)$ is length of queue. The second term represents the waiting time within the queue behind the bottleneck. We set $T_{\text {free }}=0$, hereafter. This setting will not affect the qualitative property.

The queue length that a trip maker departing at time $t$ encounters is calculated as follows:

$$
Q(t)=\max \left\{\int_{t_{0}}^{t}[r(x)-s] \mathrm{d} x, 0\right\},
$$

and the cumulative departures as,

$$
R(t)=\int_{t_{0}}^{t} r(x) \mathrm{d} x
$$

where $r(x)$ is the departure rate at time instant $t$ and $t_{0}$ is the earliest time with positive departure rate.

Suppose that every morning, a fixed number of $N$ individuals commute from home to office located in the $\mathrm{CBD}$, driving along the road stated above. All workers have identical skills and preferences. Unlike Vickrey [1] which assumes that there is only one preferred arrive time $t^{*}$, here, firms in the CBD adopt a flexi-time work schedule such that employees arriving at office earlier or later $\delta(\delta \geq 0)$ than $t^{*}$ incur no scheduling cost. Hereafter, we call this time period, $\left[t^{*}-\delta, t^{*}+\delta\right]$, as PATI.

Some commuters may still arrive at the destination earlier or later than PATI, in order to avoid a long queue at the bottleneck. The cost for commuters traveling from home to the CBD consists of three components: the cost of travel time and the cost of schedule delay early or late. It can be formulated as follows:

$$
C(t)=\alpha T(t)+\operatorname{SDE}(t)+\operatorname{SDL}(t)
$$

where $\alpha$ is the value of travel time. The cost of schedule delay early (SDE) and schedule delay late (SDL) for a commuter who leaves home at time $t$ can be expressed as

$$
\begin{aligned}
& \operatorname{SDE}(t)=\beta\left(t^{*}-\delta-(t+T(t))\right), \\
& \operatorname{SDL}(t)=\gamma\left(t+T(t)-\left(t^{*}+\delta\right)\right),
\end{aligned}
$$

where $\beta$ and $\gamma$ denote the value of schedule delay early and the value of schedule delay late, respectively. 


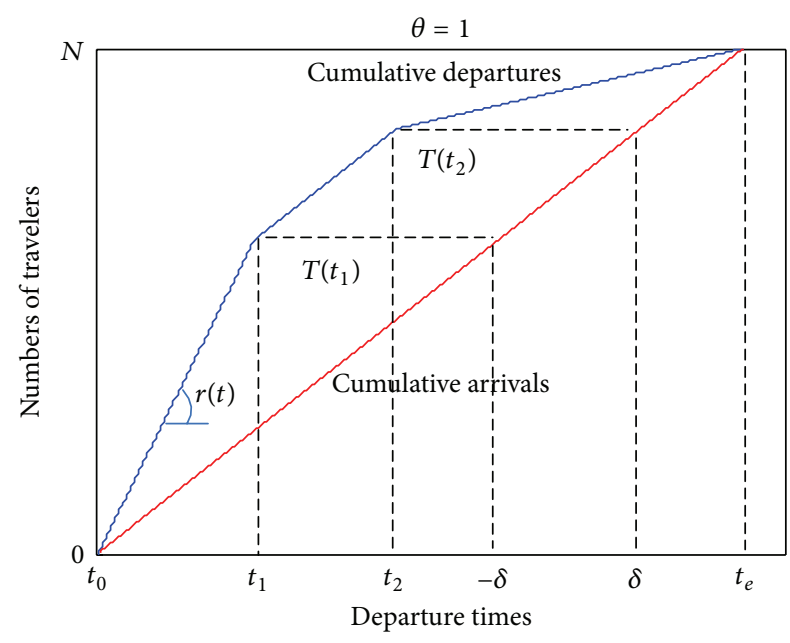

FIGURE 1: Departure time distributions in the deterministic case.

Substituting (1) and (5) into (4) and applying the equilibrium condition, $\partial C(t) / \partial t=0$, we have the equilibrium departure rate as follows,

$$
r(t)= \begin{cases}\frac{\alpha s}{(\alpha-\beta)}, & \text { if } t_{0} \leq t<t_{1} \\ s, & \text { if } t_{1} \leq t \leq t_{2} \\ \frac{\alpha s}{(\alpha+\gamma)}, & \text { if } t_{2}<t \leq t_{e},\end{cases}
$$

where $t_{0}$ and $t_{e}$ are the earliest time and the latest time with positive departure rate, respectively. And $t_{1}$ and $t_{2}$ are the watershed times for which an individual arrives at work on time. Then

$$
\begin{gathered}
t_{0}=t^{*}+\frac{\gamma-\beta}{\beta+\gamma} \delta-\frac{\gamma}{\beta+\gamma} \cdot \frac{N}{s}, \\
t_{1}=\frac{\alpha-\beta}{\alpha} t^{*}-\frac{\alpha-\beta}{\alpha} \delta-\frac{\beta \gamma}{\alpha(\beta+\gamma)} \cdot \frac{N}{s}, \\
t_{2}=\frac{\alpha-\beta}{\alpha} t^{*}+\frac{\alpha+\beta}{\alpha} \delta-\frac{\beta \gamma}{\alpha(\beta+\gamma)} \cdot \frac{N}{s}, \\
t_{e}=t^{*}+\frac{\gamma-\beta}{\beta+\gamma} \delta+\frac{\beta}{\beta+\gamma} \cdot \frac{N}{s} .
\end{gathered}
$$

Figure 1 depicts the cumulative departures (from home) and arrivals (at $\mathrm{CBD}$ ) in equilibrium under the deterministic capacity case. For simplicity, we set $t^{*}$ to be zero, and then all travelers have the same PATI $[-\delta, \delta]$. The horizontal distance between the departure and arrival curves gives the travel time. Several critical time points are indicated. Travelers departing at time interval $\left[t_{1}, t_{2}\right]$ have the longest travel time but will arrive on time, that is, within PATI. Travelers departing before $t_{1}$ will arrive earlier than desired, while the travelers departing after $t_{2}$ will arrive late at the destination.

2.2. The Stochastic Case. The deterministic case models a single-day departure time equilibrium. In the real world, road capacities may vary from day to day due to unexpected events such as incidents and weather conditions. Because of the capacity fluctuations, both commuters' travel time and their schedule delays are stochastic. In this section, we hypothesize that a constant long-term departure time pattern may emerge given the responses of the travelers to the day-to-day capacity variation. Each commuter chooses an optimal departure time which minimizes his/her long-term expected trip cost. We call this pattern, if it exists, a long-term equilibrium pattern.

2.2.1. Assumptions and Travelers' Cost Function. The following assumptions are made in the model formulation.

(A1) Commuters are homogeneous with the same $\alpha, \beta$, and $\gamma$ values and the same PATI.

(A2) The capacity of the bottleneck is constant within a day but fluctuates from day to day. The uncertainty of capacity is completely exogenous and independent of departures.

(A3) The capacity is a nonnegative stochastic variable changing around a certain mean capacity. Following Li et al. [17], we assume that stochastic capacity follows a uniform distribution within interval $[\theta \bar{s}, \bar{s}]$, where $\bar{s}$ is the design capacity and $\theta(<1)$ is a positive parameter which denotes the lowest rate of available capacity.

(A4) Commuters are aware of the capacity degeneration probability, and their departure time choice follows the user equilibrium (UE) principle in terms of mean trip cost.

We assume that the capacity of the single bottleneck is stochastic but the commuters' departure time choice is deterministic. The calculation of the mean trip cost relies on the calculations of the mean travel time, the mean schedule delay early and late. For simplicity, we set the $t^{*}$ to be zero, and then the PATI becomes $[-\delta, \delta]$. Under the stochastic condition, the mean trip cost with respect to departure time $t$ can be formulated as follows:

$$
E[c(t)]=E[\alpha T(t)+\operatorname{SDE}(t)+\operatorname{SDL}(t)] .
$$

The equilibrium condition for commuters' departure time choice in a single bottleneck with stochastic capacity is that no commuter can reduce his/her mean trip cost by unilaterally altering his/her departure time. This condition implies that the commuters' mean trip cost is fixed with respect to the time instant with positive departure rate. That is

$$
\frac{\partial E[c(t)]}{\partial t}=0, \quad \text { if } r(t)>0 .
$$

2.2.2. Mathematical Formulations and Derivations. Due to the stochastic capacity over days, the travel time experienced by a traveler departing at the same time $t$ varies from day to day. This is equivalent to saying that commuters departing home to work at the same time may endure schedule delay early/late or not and may experience queuing delay or not on different days. Consequently, there are six situations which 
may occur: (I) always arrive early, (II) possibly arrive early or on time, (III) always arrive on time, (IV) possibly arrive on time or late, (V) always arrive late, and (VI) always arrive late but queue may exist. We propose the following simple extension of trip cost function under the six situations to model user departure time choice under degradable capacities, and we use $t_{1}, t_{2}, t_{3}, t_{4}$, and $t_{5}$ to denote the watershed lines separating the six cases.

As we assume that stochastic capacity is completely exogenous and independent of departure flows, the expectation of travel time and schedule delay cost with respect to different situations can be derived respectively as follows:

$$
\begin{aligned}
E & {[T(t)] } \\
& = \begin{cases}\int_{\theta \bar{s}}^{\bar{s}}\left(\frac{R(t)}{s}+t_{0}-t\right) f(s) d s, & t_{0} \leq t \leq t_{5} \\
\int_{\theta \bar{s}}^{R(t) /\left(t-t_{0}\right)}\left(\frac{R(t)}{s}-t+t_{0}\right) f(s) d s, \quad t_{5}<t \leq t_{e},\end{cases} \\
E & {[\operatorname{SDE}(t)] } \\
& = \begin{cases}\beta \int_{\theta \bar{s}}^{\bar{s}}\left(-\delta-\frac{R(t)}{s}-t_{0}\right) f(s) d s, \\
\beta \int_{R(t) /\left(-\delta-t_{0}\right)}^{\bar{s}}\left(-\delta-\frac{R(t)}{s}-t_{0}\right) f(s) d s, & t_{1}<t \leq t_{2},\end{cases} \\
E & {[\operatorname{SDL}(t)] } \\
& = \begin{cases}\gamma \int_{\theta \bar{s}}^{R(t) /\left(\delta-t_{0}\right)}\left(\frac{R(t)}{s}+t_{0}-\delta\right) f(s) d s, & t_{3}<t \leq t_{4} \\
\gamma \int_{\theta \bar{s}}^{\bar{s}}\left(\frac{R(t)}{s}+t_{0}-\delta\right) f(s) d s,\end{cases}
\end{aligned}
$$

where $f(s)=1 /(s-\theta s)$. Substituting (10) into (8), we get the expected trip cost with respect to each situation. According to (9), the equilibrium departure rates for the six situations can be expressed as follows.

Situation 1. No commuters experience schedule delay later subject to all possible values of the bottleneck capacity. We get the departure rate

$$
r(t)=\frac{\alpha}{\alpha-\beta} \cdot \frac{\bar{s}(1-\theta)}{\ln \theta^{-1}}, \quad t_{0} \leq t<t_{1} .
$$

The boundary condition for this situation is $\operatorname{SDL}\left(t_{1}\right)=0$ when $s=\theta \bar{s}$, and hence we have $R\left(t_{1}\right)=-\left(t_{0}+\delta\right) \theta \bar{s}$.

Situation 2. If the capacity of the bottleneck is large enough, only schedule delay early will occur. On the contrary, no schedule delay occurs when the capacity is small. The watershed capacity satisfies $T(t)+t=-\delta$. Equivalently, we have $s=R(t) /\left(-\delta-t_{0}\right)$ and the departure rate,

$$
r(t)=\frac{\alpha \bar{s}(1-\theta)}{\alpha \ln \theta^{-1}-\beta\left(\ln \left(\left(-\delta-t_{0}\right) \bar{s}\right)-\ln R(t)\right)},
$$

The boundary condition for this case is $\operatorname{SDL}\left(t_{2}\right)=0$ when $s=\bar{s}$, and hence we have $R\left(t_{2}\right)=-\left(t_{0}+\delta\right) \bar{s}$.

Situation 3. No commuters experience schedule delay subject to all possible values of the bottleneck capacity. Therefore, the departure rate is

$$
r(t)=\frac{\bar{s}(1-\theta)}{\ln \theta^{-1}}, \quad t_{2}<t \leq t_{3} .
$$

The boundary condition for this case is $\operatorname{SDE}\left(t_{3}\right)=$ $\operatorname{SDL}\left(t_{3}\right)=0$ when $s=\theta \bar{s}$. Hence, we have $R\left(t_{3}\right)=\left(\delta-t_{0}\right) \theta \bar{s}$.

Situation 4. If the capacity of the bottleneck is large enough, individuals arrive on time. On the contrary, schedule delay late occurs when the capacity is small. The watershed capacity satisfies $T(t)+t=\delta$. Equivalently, we have $s=R(t) /\left(\delta-t_{0}\right)$. Therefore,

$$
r(t)=\frac{\alpha \bar{s}(1-\theta)}{\alpha \ln \theta^{-1}+\gamma\left(\ln R(t)-\ln \left(\left(\delta-t_{0}\right) \theta \bar{s}\right)\right)}, \quad t_{3}<t \leq t_{4} .
$$

The boundary condition for this case is $\operatorname{SDE}\left(t_{4}\right)=0$ when $s=\bar{s}$, and then we have $R\left(t_{4}\right)=\left(\delta-t_{0}\right) \bar{s}$.

Situation 5. Similar to Situation 1, we have

$$
r(t)=\frac{\alpha}{\alpha+\gamma} \cdot \frac{\bar{s}(1-\theta)}{\ln \theta^{-1}}, \quad t_{4}<t \leq t_{5} .
$$

The boundary condition for this case is $R\left(t_{3}\right)=\bar{s}\left(t_{5}-t_{0}\right)$; that is, the queue length at time $t_{5}$ equals to zero when $s=\bar{s}$.

Situation 6. Similar to Situation 2, we can find a watershed capacity of the bottleneck such that the queue length equals zero; that is, $R(t)=s\left(t-t_{0}\right)$, and hence the watershed capacity is $R(t) /\left(t-t_{0}\right)$. We have

$$
\begin{array}{r}
r(t)=\frac{\mathrm{d} R(t)}{\mathrm{d} t}=\frac{(\alpha+\gamma) R(t) /\left(t-t_{0}\right)-(\alpha \theta+\gamma) \bar{s}}{(\alpha+\gamma)\left(\ln R(t)-\ln \theta \bar{s}\left(t-t_{0}\right)\right)}, \\
t_{3}<t \leq t_{e} .
\end{array}
$$

The boundary condition for this case is $r\left(t_{e}\right)=0$. Equivalently, we have $R\left(t_{e}\right)=\widehat{s}\left(t_{e}-t_{0}\right)$, where $\widehat{s}=\bar{s}(\alpha \theta+$ $\gamma) /(\alpha+\gamma)$ is the mean capacity under the stochastic case.

2.2.3. Determination of the Watershed Time Instants. Since the departure rate $r(t)=0$ if $t>t_{e}$, the cumulative departures at time $t_{e}$ are equal to the traffic demand; that is, $R\left(t_{e}\right)=$ $N=\widehat{s}\left(t_{e}-t_{0}\right)$. Therefore, we have $t_{e}=t_{0}+N / \widehat{s}$. Moreover, the equilibrium condition of the stochastic bottleneck implies that $E\left[c\left(t_{0}\right)\right]=E\left[c\left(t_{e}\right)\right]$, and hence we have

$t_{0}=\frac{N}{\widehat{s}} \cdot \frac{1}{\omega_{0}-1}+\frac{v_{0}}{1-\omega_{0}} \delta, \quad t_{e}=\frac{N}{\widehat{s}} \cdot \frac{\omega_{0}}{\omega_{0}-1}+\frac{v_{0}}{1-\omega_{0}} \delta$,

where

$$
\begin{gathered}
\omega_{0}=1-\frac{(1-\theta)(\beta+\gamma)}{(\alpha \theta+\gamma)(\ln \widehat{s}-\ln \theta \bar{s})}, \\
v_{0}=\frac{(1-\theta)(\gamma-\beta)}{(\alpha \theta+\gamma)(\ln \widehat{s}-\ln \theta \bar{s})} .
\end{gathered}
$$


Using the boundary conditions of Situations 1-6, we can obtain the watershed lines as follows:

$$
\begin{gathered}
t_{1}=\omega_{1} t_{0}+v_{1} \delta, \quad t_{2}=\omega_{2} t_{0}+v_{2} \delta, \\
t_{3}=\omega_{3} t_{0}+v_{3} \delta, \quad t_{4}=\omega_{4} t_{0}+v_{4} \delta, \\
t_{5}=\omega_{5} t_{0}+v_{5} \delta,
\end{gathered}
$$

where

$$
\begin{aligned}
& \omega_{1}=1-\frac{(\alpha-\beta) \theta \xi}{\alpha}, \quad \omega_{2}=\frac{(\alpha+\beta)}{\alpha}-\xi \\
& \omega_{3}=\frac{\beta}{\alpha}-\theta \xi+1, \quad \omega_{4}=\frac{(\alpha+\gamma)(1-\xi)}{\alpha}+\frac{\beta}{\alpha}, \\
& \omega_{5}=1+\frac{(\gamma+\beta)}{(\alpha-(\alpha+\gamma) \xi)}, \quad v_{1}=\frac{(\beta-\alpha) \theta \xi}{\alpha}, \\
& v_{2}=\frac{\beta}{\alpha}-\xi, \quad v_{3}=\frac{\beta}{\alpha}+\theta \xi, \\
& v_{4}=\frac{((\alpha+\gamma) \xi+(\beta-\gamma))}{\alpha}, \quad v_{5}=\frac{(\beta-\gamma)}{(\alpha-(\alpha+\gamma) \xi)} \\
& \xi=\frac{\ln \theta^{-1}}{(1-\theta)} .
\end{aligned}
$$

With the resulting stochastic departure pattern at longterm equilibrium, the experienced day-to-day travel times and number of travelers experiencing queues change according to varied capacities over days. Given the boundary conditions of Situations 1-6, the cumulative departures and arrivals of a stochastic bottleneck are given in Figure 2. The two solid curves denote the cumulative departures and cumulative arrivals, as in Figure 1. The dotted lines are the maximum and the minimum capacity of the bottleneck, that is, $\bar{s}$ and $\theta \bar{s}$. The earliest departure time from home to workplace is $t_{0}$, and if the bottleneck capacity equals $\theta \bar{s}$, commuters departing between $t_{1}$ and $t_{3}$ will arrive at workplace on time. If the capacity equals $\bar{s}$, commuters departing between $t_{2}$ and $t_{4}$ will arrive at workplace on time. Commuters departing within time interval $\left[t_{2}, t_{3}\right]$ will always arrive at workplace on time, whatever the variability of capacity is. At the beginning, commuters depart from home with a constant departure rate until the watershed time $t_{1}$. Subsequently, the departure rate will gradually drop down. After time instant $t_{4}$, another constant departure rate will last until the watershed time instant $t_{5}$ and the queuing length at this time will be zero. Later, the departure rate continues to drop down to zero at time $t_{e}$.

2.2.4. Properties of the Stochastic Bottleneck Model. In this subsection, we investigate the theoretical properties of the equilibrium solution of the proposed stochastic bottleneck model with PATI.

Theorem 1. At equilibrium, the expected trip cost for all commuters is a monotonically increasing function of traffic demand and a monotonically decreasing function of $\delta$-value; that is, $\partial E\left[c\left(t_{0}\right)\right] / \partial N>0$ and $\partial E\left[c\left(t_{0}\right)\right] / \partial \delta<0$ hold.

Proof. Since $E\left[c\left(t_{0}\right)\right]=\left(-\delta-t_{0}\right) \beta$ and $t_{0}=N /\left(\widehat{s}\left(\omega_{0}-1\right)\right)+$ $v_{0} \delta /\left(1-\omega_{0}\right)$, we have $\partial E\left[c\left(t_{0}\right)\right] / \partial \delta=-2 \beta \gamma /(\beta+\gamma)<0$ and

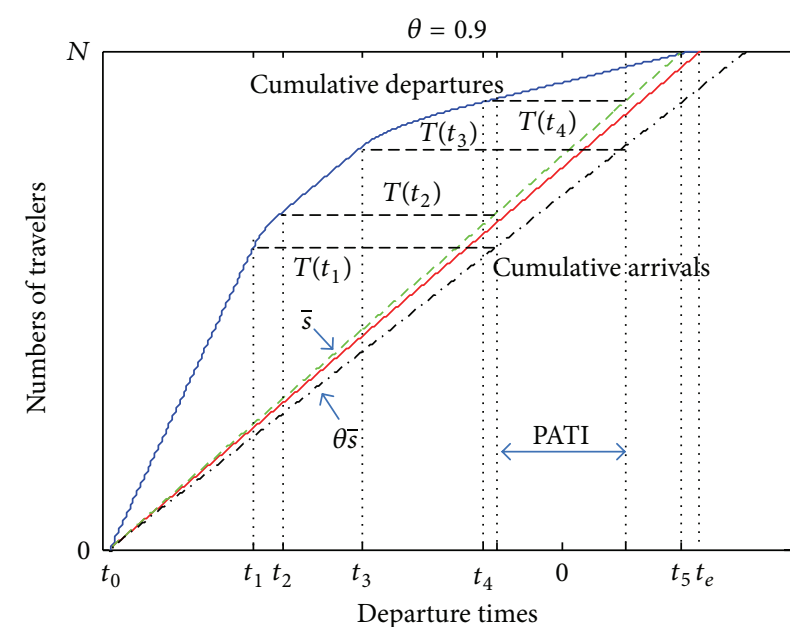

Figure 2: Departure time distributions in the stochastic case.

$\partial E\left[c\left(t_{0}\right)\right] / \partial N=\beta /\left(\left(1-\omega_{0}\right) \widehat{s}\right)$. The definitions of $\beta$ and $\widehat{s}$ imply that they are positive; hence, to prove $\partial E\left[c\left(t_{0}\right)\right] / \partial N>0$, we only need to prove $1-\omega_{0}>0$. Since $0<\theta<1$ and $\widehat{s}=\bar{s}(\alpha \theta+$ $\gamma) /(\alpha+\gamma)>\bar{s}(\alpha \theta+\gamma \theta) /(\alpha+\gamma)=\theta \bar{s}$, we have $1-\theta>0$. And $\ln \widehat{s}-\ln \theta \bar{s}>0$ clearly holds. Therefore, both the numerator and denominator of the second term in the right-hand side of the first equation of (18) are positive; therefore, we have $1-\omega_{0}>0$. This completes the proof.

Theorem 2. At equilibrium, the expected trip cost for all commuters is a monotonically decreasing function of parameter $\theta$-value; that is, $\partial E\left[c\left(t_{0}\right)\right] / \partial \theta<0$ holds.

Proof. Submitting $t_{0}=N /\left(\widehat{s}\left(\omega_{0}-1\right)\right)+v_{0} \delta /\left(1-\omega_{0}\right)$ into $E\left[c\left(t_{0}\right)\right]=\left(-\delta-t_{0}\right) \beta$, then the first-order derivative can be given as follows:

$$
\frac{\partial E[c]}{\partial \theta}=\beta \frac{N}{\bar{s}} \cdot \frac{\alpha+\gamma}{\beta+\gamma} p^{\prime}(\theta),
$$

where

$$
\begin{gathered}
p(\theta)=\frac{\ln \widehat{s}-\ln (\theta \bar{s})}{1-\theta} \\
p^{\prime}(\theta)=\frac{1}{1-\theta}\left(p(\theta)-\frac{\gamma}{\theta(\alpha \theta+\gamma)}\right) .
\end{gathered}
$$

Since $\ln (\widehat{s} /(\theta \bar{s}))<1-\theta \bar{s} / \widehat{s}=\gamma(1-\theta) /(\alpha \theta+\gamma)$, we then have

$$
\begin{aligned}
p(\theta) & -\frac{\gamma}{\theta(\alpha \theta+\gamma)} \\
& <\frac{\gamma}{\alpha \theta+\gamma}-\frac{\gamma}{\theta(\alpha \theta+\gamma)}=\frac{\gamma}{\alpha \theta+\gamma}\left(1-\frac{1}{\theta}\right)<0 .
\end{aligned}
$$

It is clear that $p^{\prime}(\theta)<0$ and therefore we get $\partial E[c] / \partial \theta<0$. This completes the proof.

Theorem 3. At equilibrium, the departure rate is a monotonically decreasing function of the departure time throughout the whole peak period; that is, $\mathrm{d} r(t) / \mathrm{d} t \leq 0, t \in\left[t_{0}, t_{e}\right]$. 
Proof. According to (11)-(16), the departure rate $r(t)$ is continuous within each of intervals $\left[t_{0}, t_{1}\right),\left(t_{1}, t_{2}\right),\left(t_{2}, t_{3}\right)$, $\left(t_{3}, t_{4}\right),\left(t_{4}, t_{5}\right)$, and $\left(t_{5}, t_{e}\right]$. To prove the departure rate is continuous during the peak period, we calculate the following limitations:

$$
\begin{aligned}
\lim _{t \rightarrow t_{1}^{+}} r(t) & =\lim _{t \rightarrow t_{1}^{+}} \frac{\alpha \bar{s}(1-\theta)}{\alpha \ln \theta^{-1}-\beta\left(\ln \left(\left(-\delta-t_{0}\right) \bar{s}\right)-\ln R(t)\right)} \\
& =\frac{\alpha}{\alpha-\beta} \cdot \frac{\bar{s}(1-\theta)}{\ln \theta^{-1}}=\lim _{t \rightarrow t_{1}^{-}} r(t), \\
\lim _{t \rightarrow t_{2}^{-}} r(t) & =\frac{\alpha \bar{s}(1-\theta)}{\alpha \ln \theta^{-1}-\beta\left(\ln \left(\left(-\delta-t_{0}\right) \bar{s}\right)-\ln R(t)\right)} \\
& =\frac{\bar{s}(1-\theta)}{\ln \theta^{-1}}=\lim _{t \rightarrow t_{2}^{+}} r(t), \\
\lim _{t \rightarrow t_{3}^{-}} r(t) & =\frac{\bar{s}(1-\theta)}{\ln \theta^{-1}} \\
& =\frac{\alpha \bar{s}(1-\theta)}{\alpha \ln \theta^{-1}+\gamma\left(\ln R(t)-\ln \left(\left(\delta-t_{0}\right) \theta \bar{s}\right)\right)} \\
& =\lim _{t \rightarrow t_{3}^{+}} r(t), \\
\lim _{t \rightarrow t_{4}^{-}} r(t) & =\frac{\alpha \bar{s}(1-\theta)}{\alpha \ln \theta^{-1}+\gamma\left(\ln R(t)-\ln \left(\left(\delta-t_{0}\right) \theta \bar{s}\right)\right)} \\
& =\frac{\alpha}{\alpha+\gamma} \cdot \frac{\bar{s}(1-\theta)}{\ln \theta^{-1}}=\lim _{t \rightarrow t_{4}^{+}} r(t), \\
\lim _{t \rightarrow t_{5}^{+}} r(t) & =\frac{(\alpha+\gamma) R\left(t_{3}\right) /\left(t_{3}-t_{0}\right)-(\alpha \theta+\gamma) \bar{s}}{(\alpha+\gamma) \ln \left(R\left(t_{3}\right) /\left(\theta \bar{s}\left(t_{3}-t_{0}\right)\right)\right)} \\
& =\frac{\alpha}{\alpha+\gamma} \cdot \frac{\bar{s}(1-\theta)}{\ln \theta^{-1}=\lim _{t \rightarrow t_{5}^{-}} r(t) .}
\end{aligned}
$$

This proves $r(t)$ is continuous indeed within the interval $\left[t_{0}, t_{e}\right]$.

Equations (11), (13), and (15) state that the departure rate $r(t)$ is constant for $t_{0} \leq t \leq t_{1}, t_{2} \leq t \leq t_{3}$, and $t_{4} \leq t \leq$ $t_{5}$, hence it is monotonically decreasing within these three intervals. By definition, the cumulative departure flow $R(t)$ is nondecreasing with respect to time $t$. Thus, the denominators of the right-hand sides in (12) and (14) are nondecreasing with respect to time $t$. Therefore, the right-hand sides of (12) and (14) are nonincreasing with respect to time $t$; that is, the departure rate $r(t)$ is monotonically decreasing within $\left[t_{1}, t_{2}\right]$ and $\left[t_{3}, t_{4}\right]$. The proof of $\mathrm{d} r(t) / \mathrm{d} t \leq 0$ for all $t \in\left(t_{5}, t_{e}\right]$ can be found in Xiao et al. [18].

In summary, the departure rate $r(t)$ is monotonically decreasing within all four intervals and at their boundaries. Considering the continuity of $r(t)$ for all $t \in\left[t_{0}, t_{e}\right]$, we conclude that $r(t)$ is monotonically decreasing within $\left[t_{0}, t_{e}\right]$. This completes the proof.

Proposition 4. When parameter $\theta$ approaches one, the stochastic bottleneck model immediately follows the deterministic model.
Proof. According to the L'Hôpital's rule, we have $\lim _{\theta \rightarrow 1}(1-$ $\theta) /\left(\ln \theta^{-1}\right)=1$. We then have

$$
\begin{gathered}
\lim _{\theta \rightarrow 1} \omega_{0}=\lim _{\theta \rightarrow 1} \omega_{5}=\frac{-\beta}{\gamma}, \\
\lim _{\theta \rightarrow 1} \omega_{1}=\lim _{\theta \rightarrow 1} \omega_{2}=\lim _{\theta \rightarrow 1} \omega_{3}=\lim _{\theta \rightarrow 1} \omega_{4}=\frac{\beta}{\alpha}, \\
\lim _{\theta \rightarrow 1} v_{0}=\lim _{\theta \rightarrow 1} v_{5}=\frac{(\gamma-\beta)}{\gamma}, \\
\lim _{\theta \rightarrow 1} v_{1}=\lim _{\theta \rightarrow 1} v_{2}=\frac{(\beta-\alpha)}{\alpha}, \quad \lim _{\theta \rightarrow 1} v_{3}=\lim _{\theta \rightarrow 1} v_{4}=\frac{(\beta+\alpha)}{\alpha}, \\
\lim _{\theta \rightarrow 1} r(t)= \begin{cases}\frac{\alpha \bar{s}}{(\alpha-\beta)}, & \text { if } t_{0} \leq t \leq t_{1} \\
\bar{s}, & \text { if } t_{1} \leq t \leq t_{4} \\
\frac{\alpha \bar{s}}{(\alpha+\gamma)}, & \text { if } t_{4}<t \leq t_{e} .\end{cases}
\end{gathered}
$$

Substituting (26) into (19), we have

$$
\begin{gathered}
t_{0}=-\frac{\gamma}{\beta+\gamma} \cdot \frac{N}{\bar{s}}+\frac{\gamma-\beta}{\beta+\gamma} \delta, \quad t_{5}=t_{e}=\frac{\beta}{\beta+\gamma} \cdot \frac{N}{\bar{s}}+\frac{\gamma-\beta}{\beta+\gamma} \delta, \\
t_{1}=t_{2}=-\frac{\beta \gamma}{\alpha(\beta+\gamma)} \cdot \frac{N}{s}+\frac{\beta-\alpha}{\alpha} \delta, \\
t_{3}=t_{4}=-\frac{\beta \gamma}{\alpha(\beta+\gamma)} \cdot \frac{N}{s}+\frac{\alpha+\beta}{\alpha} \delta .
\end{gathered}
$$

Therefore, we get the same traffic flow pattern with that from a deterministic bottleneck model.

Proposition 5. When the number of commuters is given, increasing the value of parameter $\theta$ will result in a decrease in the length of peak period.

Proof. According to (16), we have $\mathrm{d} \widehat{s} / \mathrm{d} \theta=\alpha /(\alpha+\gamma)>0$. This implies that $\widehat{s}$ is a monotonic increasing function with respect to $\theta$. According to (17), we can obtain the length of peak period as follows:

$$
t_{e}-t_{0}=\frac{N}{\widehat{s}} \frac{\omega_{0}}{\omega_{0}-1}+\frac{v_{0}}{1-\omega_{0}} \delta-\frac{N}{\widehat{s}} \frac{1}{\omega_{0}-1}-\frac{v_{0}}{1-\omega_{0}} \delta=\frac{N}{\widehat{s}} .
$$

Since $N$ is constant and $\widehat{s}$ is a monotonic increasing function with respect to $\theta, t_{e}-t_{0}$ is also a monotonic increasing function with respect to $\theta$. This completes the proof.

The above proof also shows that the length of peak period is not affected by the $\delta$-value.

\section{Numerical Examples}

The input parameters of our numerical example are $\alpha=$ $6.4 \$ / \mathrm{h}, \beta=3.9 \$ / \mathrm{h}, \gamma=15.21 \$ / \mathrm{h}, N=6000 \mathrm{veh}, \bar{s}=$ $4000 \mathrm{veh} / \mathrm{h}, \delta=10 \mathrm{~min}$, and $\theta=0.9$. By solving the proposed 
TABLE 1: The influence of parameter $\theta$ on the mean trip cost and the watershed time instant.

\begin{tabular}{lccccccccc}
\hline$\theta$ & $E[c(t)]$ & $t_{0}$ & $t_{1}$ & $t_{2}$ & $t_{3}$ & $t_{4}$ & $t_{5}$ & $t_{e}$ & $t_{e}-t_{0}$ \\
\hline 1.00 & 3.62 & -1.10 & -0.74 & -0.74 & -0.40 & -0.40 & 0.40 & 0.40 & 1.50 \\
0.95 & 3.78 & -1.14 & -0.77 & -0.73 & -0.46 & -0.31 & 0.36 & 0.39 \\
0.90 & 3.95 & -1.18 & -0.80 & -0.73 & -0.52 & -0.21 & 0.31 & 0.37 \\
0.85 & 4.13 & -1.23 & -0.85 & -0.72 & -0.59 & -0.09 & 0.26 & 0.35 & 1.52 \\
0.80 & 4.33 & -1.28 & -0.89 & -0.71 & -0.66 & 0.05 & 0.21 & 0.32 & 1.57 \\
\hline
\end{tabular}

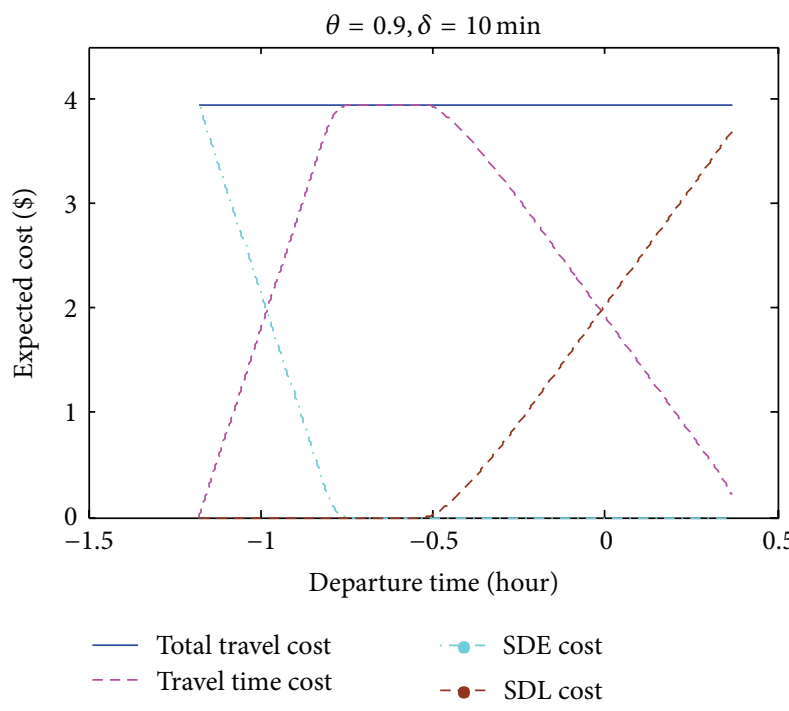

FIgURE 3: The mean equilibrium trip cost and other components.

model, we obtained the time period with positive departure rate during $[-1.18,0.37]$ (see Table 1$)$. The mean trip cost, the mean travel time cost, and the mean schedule delay cost (SDE and SDL) are depicted in Figure 3. We find that the mean trip costs of all commuters are the same and are equal to $3.95 \$$, but endure a trade-off between cost of travel time and cost of schedule delay. It is interesting that the waiting time cost is not zero at the end of the peak period, indicating that queue still exists.

It is interesting to investigate the impact of the fraction parameter $\theta$ on the solution of the stochastic bottleneck model. We changed the parameter $\theta$ from 0.8 to 1.0 , and computed the mean trip cost and the watershed time instants. The results are shown in Table 1; the row highlighted is with the default $\theta$-value. It can be seen that $t_{1}=t_{2}, t_{3}=t_{4}$, and $t_{5}=$ $t_{e}$ when $\theta=1.0$. This confirms Proposition 4 . We can also find that the length of time period with positive departure rate increases with the $\theta$-value. This confirms Proposition 5. In addition, the second column of Table 1 shows that the mean trip cost decreases with the increase of $\theta$-value. This confirms Theorem 2. Since decreasing the $\theta$-value is equivalent to increasing the travel time uncertainty, commuters will leave home earlier than before for avoiding the potential loss caused by uncertainty risk.

The departure rates against different $\theta$-values are displayed in Figure 4. It can be clearly seen that the results confirm Propositions 4 and 5 that the stochastic bottleneck model immediately follows the deterministic model when

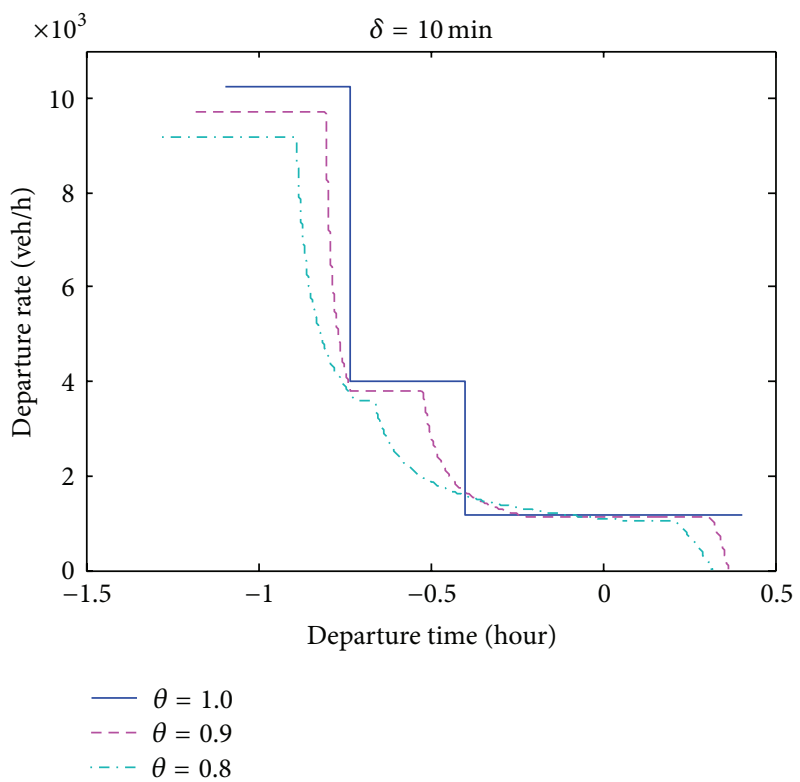

Figure 4: The influence of parameter $\theta$ on departure rate.

$\theta$ approaches one, and that enlarging the parameter $\theta$ will result in a decrease in the length of peak period. Figure 4 also shows that in equilibrium the departure rate during the peak period is monotonically decreasing, which is consistent with Theorem 3.

Table 2 lists the mean trip costs and the watershed time instants when $\theta=0.9$ and $\delta$-value from 0 minutes to 20 minutes. It is shown that in equilibrium the mean trip cost is monotonically decreasing with increasing $\delta$, whilst the length of the peak period remains unchanged. This is consistent with Theorem 1 .

Figure 5 depicts the departure rates against different $\delta$ values at $\theta=0.9$. It can be seen that with larger $\delta$-value (or longer PATI), the peak periods shift to later and the amount of earlier departures (at a rate higher than capacity) reduces. The overall departure time patterns become more flat and approach designed capacity of the bottleneck with increasing $\delta$-value. This suggests that traffic congestion can be alleviated by adopting flexi-time work schedule, similar to that achieved through congestion pricing policy (see, Arnott et al. [5]).

Figure 6 depicts the mean trip times with different preferred arrival time intervals when $\theta=0.9$. One can observe from this figure that adopting flexi-time work schedule can reduce the commuters' travel time or the queue behind the bottleneck. The areas below three curves are 0.6208, 0.5940, and 0.5131 hours, respectively. It shows further that the 
TABLE 2: The influence of parameter $\delta$ on the mean trip cost and the watershed time instants.

\begin{tabular}{lccccccccc}
\hline$\delta$ min & $E[c(t)]$ & $t_{0}$ & $t_{1}$ & $t_{2}$ & $t_{3}$ & $t_{4}$ & $t_{5}$ & $t_{e}$ & $t_{e}-t_{0}$ \\
\hline 0 & 4.98 & -1.28 & -0.80 & -0.80 & -0.80 & -0.55 & 0.21 & 0.27 & 1.55 \\
5 & 4.46 & -1.23 & -0.80 & -0.72 & -0.68 & -0.38 & 0.26 & 0.32 & 1.55 \\
10 & 3.95 & -1.18 & -0.80 & -0.73 & -0.52 & -0.21 & 0.31 & 0.37 \\
15 & 3.43 & -1.13 & -0.80 & -0.74 & -0.36 & -0.04 & 0.36 & 0.42 & 1.55 \\
20 & 2.91 & -1.08 & -0.80 & -0.75 & -0.20 & 0.13 & 0.41 & 0.47 \\
\hline
\end{tabular}

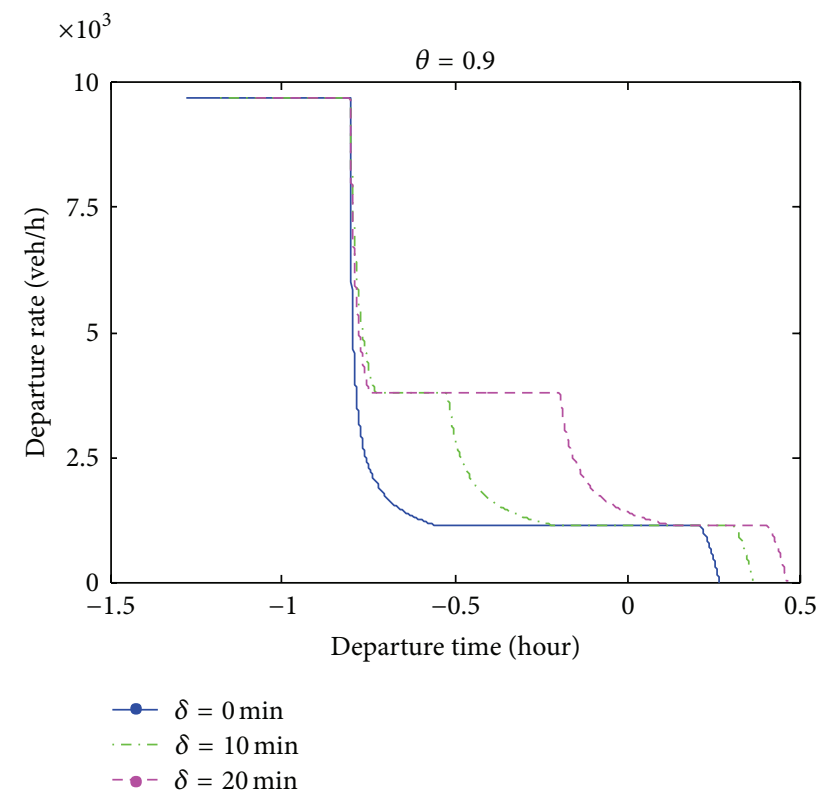

FIGURE 5: The influence of parameter $\delta$ on departure rate.

adoption of flexi-time in equilibrium leads to less congestion than under the fixed work schedule.

Figure 7 shows the joint effect of the $\theta$-value and the $\delta$-value on the equilibrium trip cost. For a fixed $\theta$, the expected trip cost declines with an increase of the preferred arrival time interval. Since the schedule delay costs endured by commuters are reduced with an increase of the length PATI without changing the departure pattern, this means that transport policies to encourage firms in CBD to adopt flexi-time can ease overall system traffic congestion. On the other hand, for a fixed $\delta$-value, the expected trip cost declines with the decrease of $\theta$-value, which confirms that improving system reliability and reducing uncertainty will increase the system's effectiveness.

\section{Conclusion}

This paper investigated the travel choice behavior under uncertainty on morning commute problem by considering the capacity variability of a highway bottleneck. The bottleneck model was applied to analyze the departure time pattern of a group of homogeneous commuters with the same preferred arrival time interval. The capacity of the bottleneck is assumed to follow a uniform distribution and

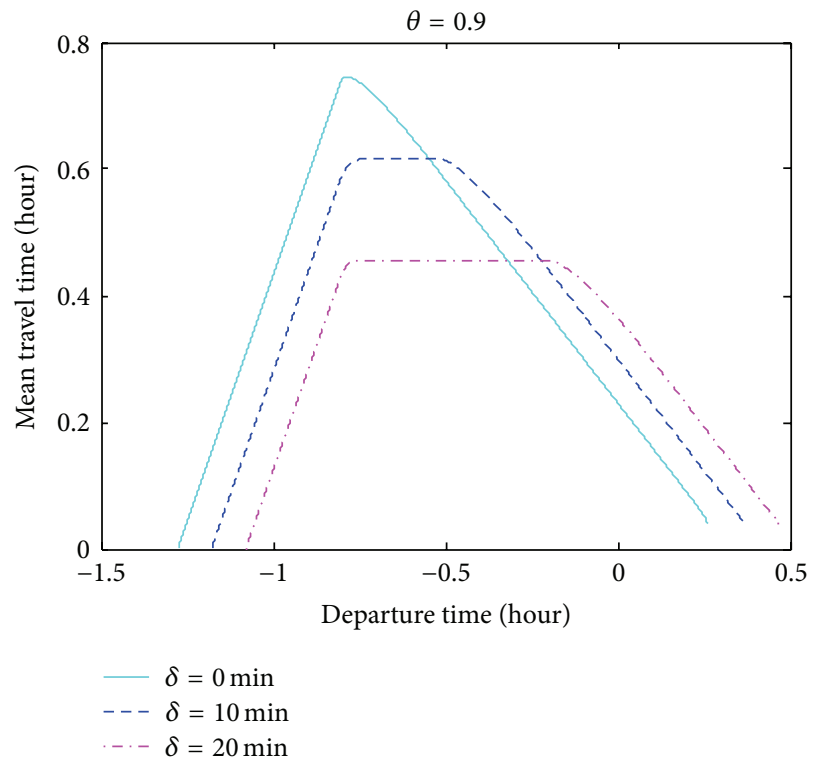

Figure 6: Travel time with different $\delta$-value.

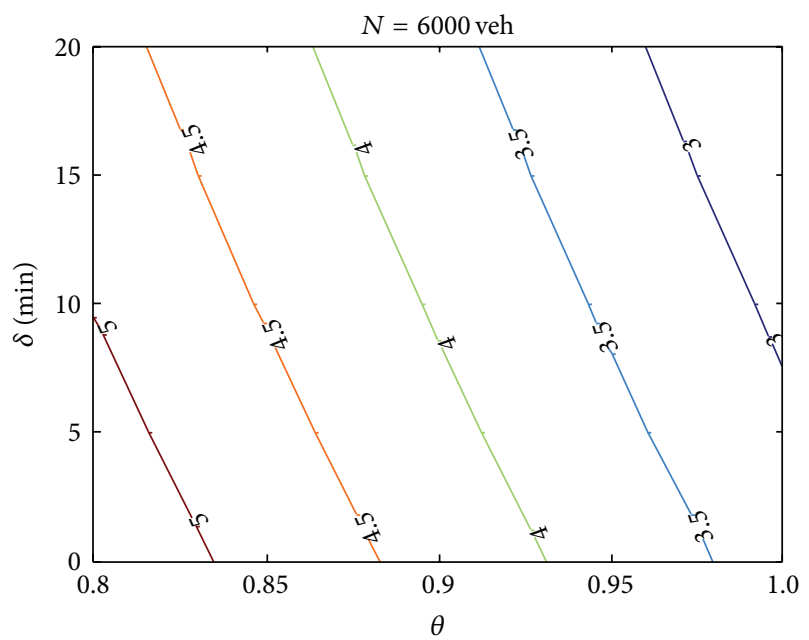

Figure 7: The equilibrium trip cost $E[C]$ with different $\theta$ and $\delta$.

the commuters' departure time choice to follow the UE principle in terms of the mean trip cost. The analytical solution of the stochastic bottleneck model was derived. Both analytical and numerical results show that increasing the capacity variation results in longer peak period and higher commuters' mean trip cost. In addition, it is shown that with longer flexi-time interval, the departure time distributions 
become flatter. This suggests that flexi-time is an effective demand management measure for alleviating peak congestion. For future research, we will further improve the model with consideration of heterogeneous commuters and travel risk and apply the model in analyzing such policy measures as congestion pricing, metering, and flexible work scheme.

\section{Conflict of Interests}

The authors declare that there is no conflict of interests regarding the publication of this paper.

\section{Acknowledgments}

The authors acknowledge thank the financial support from the National Basic Research Program of China (2012CB725401), the PhD Student Innovation Fund of Beihang University (302976), and the China Scholarship Council.

\section{References}

[1] W. S. Vickrey, "Congestion theory and transport investment," American Economic Review, vol. 59, pp. 251-261, 1969.

[2] M. J. Smith, "The existence of a time-dependent equilibrium distribution of arrivals at a single bottleneck," Transportation Science, vol. 18, no. 4, pp. 385-394, 1984.

[3] C. F. Daganzo, "Uniqueness of a time-dependent equilibrium distribution of arrivals at a single bottleneck," Transportation Science, vol. 19, no. 1, pp. 29-37, 1985.

[4] R. M. Braid, "Uniform versus peak-load pricing of a bottleneck with elastic demand," Journal of Urban Economics, vol. 26, no. 3, pp. 320-327, 1989.

[5] R. Arnott, A. de Palma, and R. Lindsey, "Economics of a bottleneck," Journal of Urban Economics, vol. 27, no. 1, pp. 111-130, 1990.

[6] H. Yang and H.-J. Huang, "The multi-class, multi-criteria traffic network equilibrium and systems optimum problem," Transportation Research Part B, vol. 38, no. 1, pp. 1-15, 2004.

[7] G. Ramadurai, S. V. Ukkusuri, J. Zhao, and J.-S. Pang, "Linear complementarity formulation for single bottleneck model with heterogeneous commuters," Transportation Research Part B, vol. 44, no. 2, pp. 193-214, 2010.

[8] M. J. Machina, "Choice under uncertainty: problem solved and unsolved," Journal of Economic Perspectives, vol. 1, no. 1, pp. 121$154,1987$.

[9] R. P. Cubitt and R. Sugden, "Dynamic decision-making under uncertainty: an experimental investigation of choice between accumulator gambles," Journal of Risk and Uncertainty, vol. 22, no. 2, pp. 103-128, 2001.

[10] C. Gollier and N. Treich, "Decision-making under scientific uncertainty: the economics of the precautionary principle," Journal of Risk and Uncertainty, vol. 27, no. 1, pp. 77-103, 2003.

[11] M. H. Birnbaum and U. Schmidt, "An experimental investigation of violations of transitivity in choice under uncertainty," Journal of Risk and Uncertainty, vol. 37, no. 1, pp. 77-91, 2008.

[12] C. Starmer, "Developments in non-expected utility theory: the hunt for a descriptive theory of choice under risk," Journal of Economic Literature, vol. 38, no. 2, pp. 332-382, 2000.
[13] A. de Palma, M. Ben-Akiva, D. Brownstone et al., "Risk, uncertainty and discrete choice models," Marketing Letters, vol. 19, no. 3-4, pp. 269-285, 2008.

[14] J. V. Henderson, "The economics of staggered work hours," Journal of Urban Economics, vol. 9, no. 3, pp. 349-364, 1981.

[15] S.-I. Mun and M. Yonekawa, "Flextime, traffic congestion and urban productivity," Journal of Transport Economics and Policy, vol. 40, no. 3, pp. 329-358, 2006.

[16] B. Siu and H. K. Lo, "Equilibrium trip scheduling in congested traffic under uncertainty," in Proceedings of the 18th International Symposium on Transportation and Traffic Theory, W. H. K. Lam, S. C. Wong, and H. K. Lo, Eds., pp. 19-38, Elsevier, Oxford, UK, 2009.

[17] H. Li, M. Bliemer, and P. Bovy, "Departure time distribution in the stochastic bottleneck model," International Journal of ITS Research, vol. 6, no. 2, pp. 79-86, 2008.

[18] L. L. Xiao, H. J. Huang, and R. Liu, "Congestion behavior and tolls in a bottleneck model with stochastic capacity," Transportation Science, 2013.

[19] A. Chen, Z. Ji, and W. Recker, "Travel time reliability with risksensitive travelers," Transportation Research Record, no. 1783, pp. 27-33, 2002. 


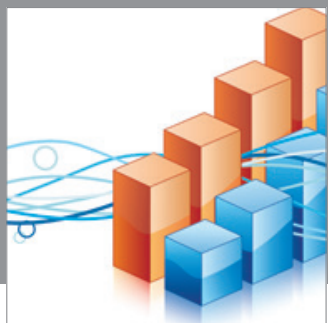

Advances in

Operations Research

mansans

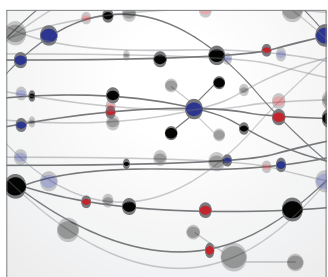

The Scientific World Journal
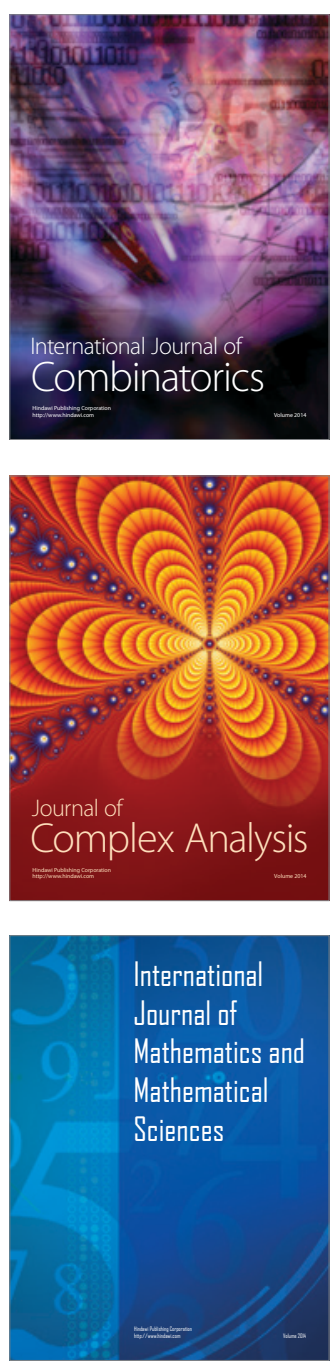
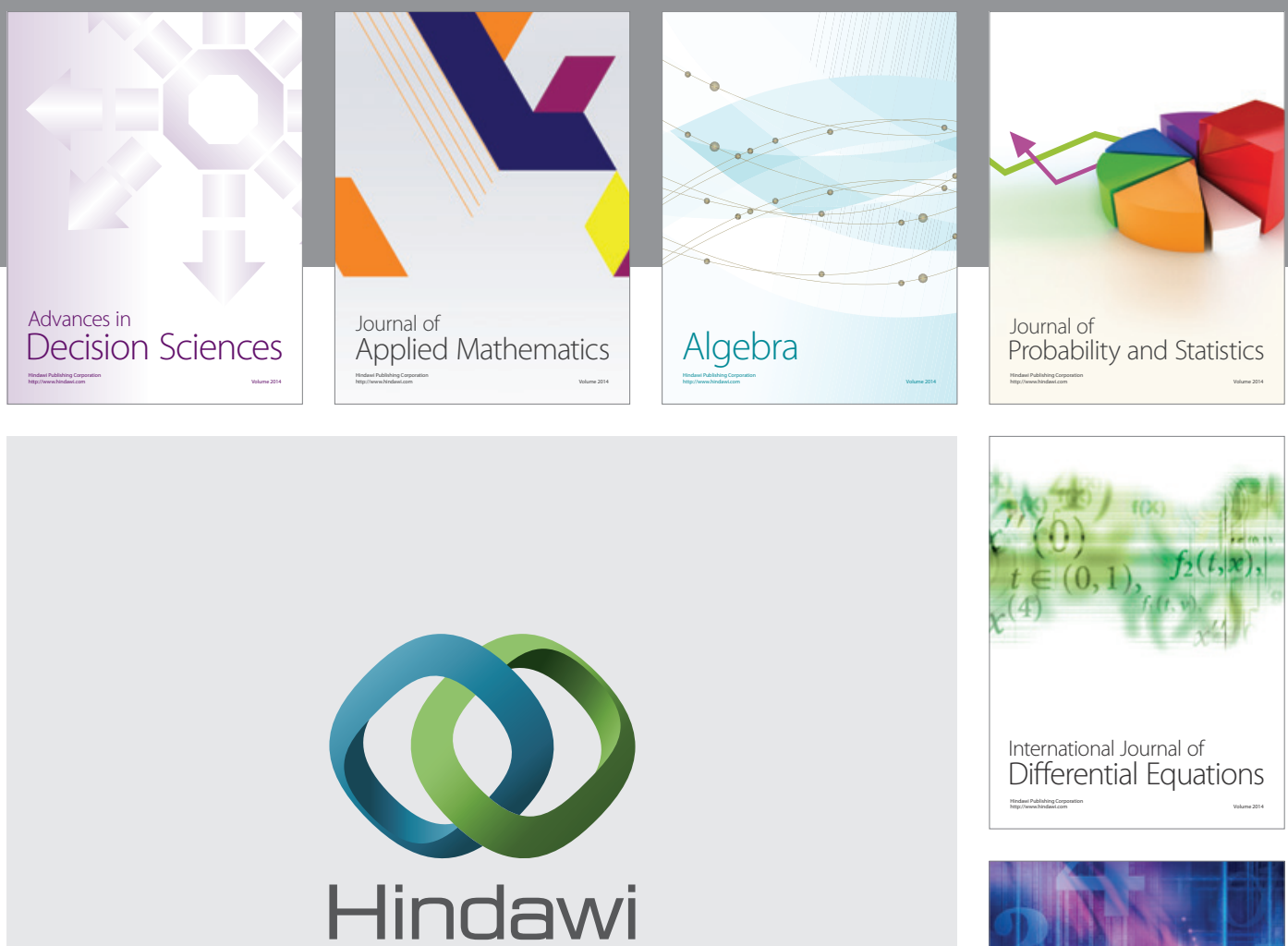

Submit your manuscripts at http://www.hindawi.com
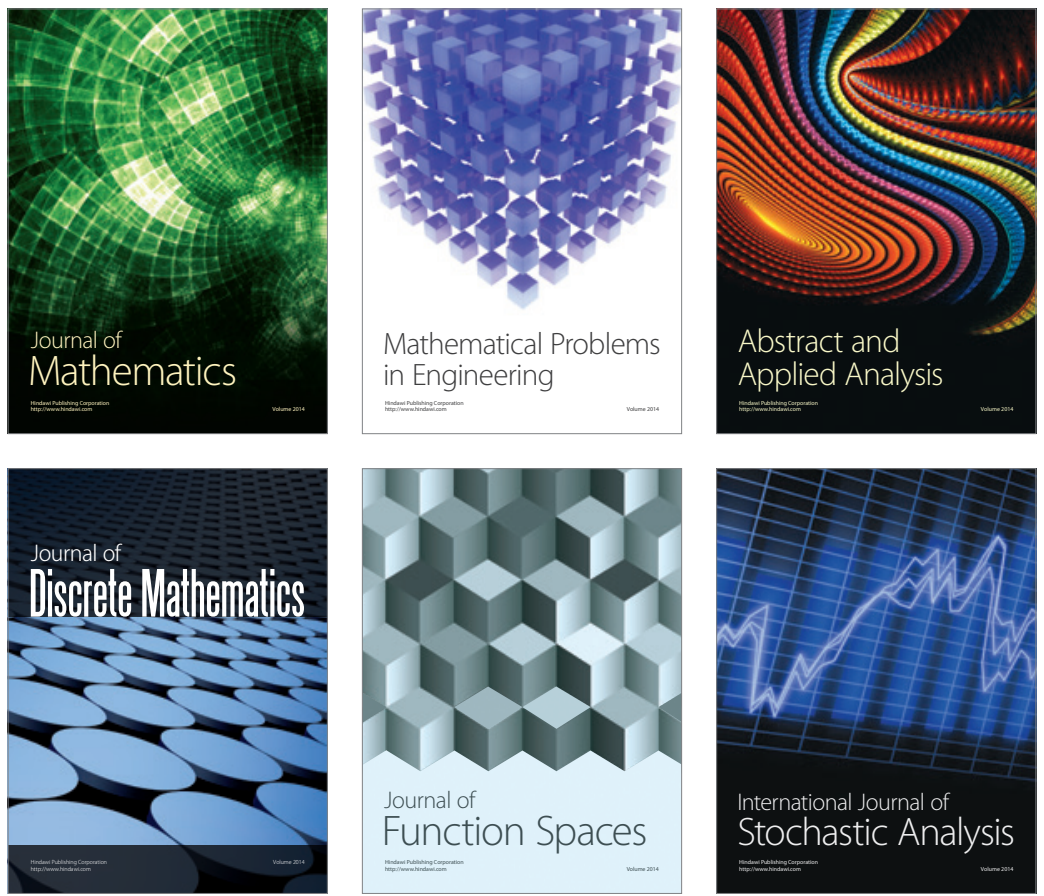

Journal of

Function Spaces

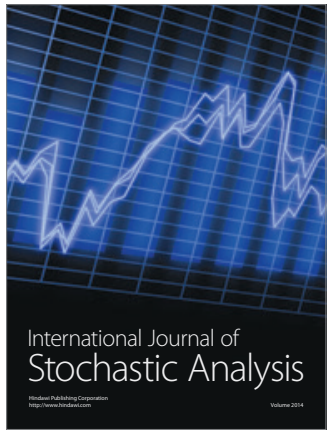

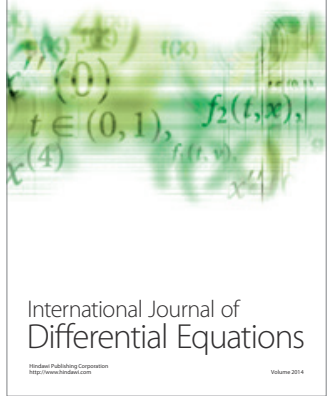
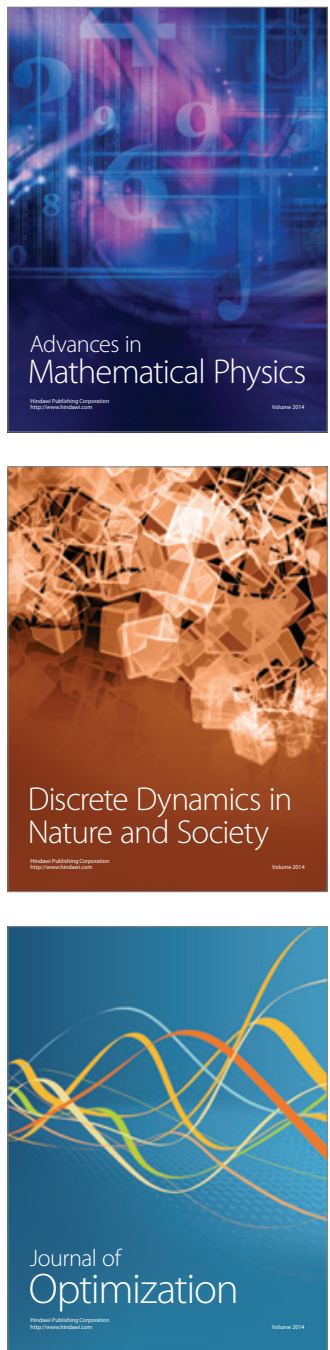